\title{
Distribución y abundancia del cardenal amarillo [Gubernatrix cristata, Passeriformes: Thraupidae] en el sur de la provincia de Buenos Aires
}

\author{
Distribution and Abundance of Yellow cardinal (Gubernatrix \\ cristata, Passeriformes: Thraupidae) in South Buenos Aires \\ Province
}

D.O.I.: https://doi.org/10.30550/j.azl/2018.62.2/4

\author{
Germán Marateo ${ }^{1,2 *}$, Diego Archuby ${ }^{1,2}$, Fabrizio Piantanida ${ }^{3}$, \\ Martín Sotelo ${ }^{3}$, Fernando Segura ${ }^{4}$ \\ 1 Dirección de Recursos Naturales - Organismo Provincial para el Desarrollo Sostenible (OPDS). Provincia \\ de Buenos Aires, Argentina. Calle 12 y 54, Torre Gubernamental II. La Plata, Argentina. \\ * gmarateo@yahoo.com \\ 2 Facultad de Ciencias Naturales y Museo, Universidad Nacional de La Plata, Calle 122 y 60, La Plata, \\ Argentina. \\ 3 Dirección de Áreas Naturales Protegidas - Organismo Provincial para el Desarrollo Sostenible (OPDS). \\ Provincia de Buenos Aires. Calle 12 y 54, Torre Gubernamental II. La Plata, Argentina. \\ 4 Club de Observadores de Aves (COA) Necochea. Calle 61 No 4535. Necochea, Argentina.
}

\begin{abstract}
> Resumen - El Cardenal amarillo (Gubernatrix cristata) es una especie "En Peligro", distribuída principalmente en el Espinal sudamericano. En la provincia de Buenos Aires se distribuye principalmente en los partidos de Bahía Blanca, Puan, Villarino y Patagones. El conocimiento sobre su distribución actual, el estado de sus poblaciones y el uso de hábitat es insuficiente. El presente trabajo realiza una aproximación al conocimiento del estado actual de las poblaciones del Cardenal amarillo en el sur de Buenos Aires, aportando nuevos datos sobre su distribución, abundancia y sobre el uso del bosque. Llevamos a cabo 303 puntos de muestreo extensivos e independientes, de radio ilimitado, con la utilización de "playback", sobre 13 transectas, totalizando $1.183 \mathrm{~km}$ entre 2015 y 2017, en 11 campañas de muestreo. Se estimó el porcentaje de cobertura de bosque en cada punto en un radio de 100 $\mathrm{m}$. Se obtuvieron 19 registros con un total de 48 individuos: 24 machos, 17 hembras, y 7 individuos indeterminados. El 78,9 \% de los registros y el $83,3 \%$ de los individuos fueron observados en la zona Sur. La cobertura del bosque fue similar entre sitios con y sin registros de la especie. Los escasos registros obtenidos en este estudio en las zonas Norte y Centro podrían deberse a la captura ilegal de ejemplares, siempre que la especie haya sido abundante en el pasado, pero no buscada; de lo contario la especie podría haber sido siempre escasa en estas zonas.
\end{abstract}

Palabras clave: Aves amenazadas, abundancia poblacional, uso del bosque, Espinal y Monte.

\footnotetext{
> Ref. bibliográfica: Marateo, G.; Archuby, D.; Piantanida, F.; Sotelo, M.; Segura, F. 2018. Distribución y abundancia del cardenal amarillo (Gubernatrix cristata, Passeriformes: Thraupidae) en el sur de la provincia de Buenos Aires. Acta zoológica lilloana 62 (2): 31-43.
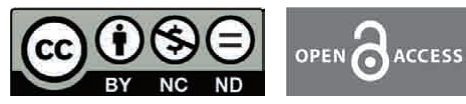

- Recibido: 19/07/18 - Aceptado: 12/11/18

$>$ URL de la revista: http://actazoologica.lillo.org.ar

- Algunos derechos reservados. Esta obra está bajo una Licencia

Creative Commons Atribución - No Comercial - Sin Obra Derivada 4.0 Internacional.
} 
> Abstract - The Yellow Cardinal (Gubernatrix cristata) is an endangered species distributed mainly in the South American Espinal. In Buenos Aires province it is mainly distributed in the departments of Bahía Blanca, Puan, Villarino and Patagones. The knowledge about its current distribution, population status and habitat use is currently scarce. This work makes an approximation to the knowledge of the current status of the populations of the species in the south of Buenos Aires, contributing new data on its distribution, abundance and on forest use. We performed 303 independent extensive, unlimited, radio sampling points with the use of playback over 13 transects, totaling 1,183 km between 2015 and 2017, during 11 sampling campaigns. The percentage of forest cover was estimated at each point within $100 \mathrm{~m}$ radius. We obtained 19 records of a total of 48 individuals: 24 males, 17 females, and 7 undeterminated individuals. The $78,9 \%$ of records and the $83,3 \%$ of individuals were observed in the southern area. Forest cover was similar between sites with and without Yellow Cardinal records. The low records at the North and Centre areas could be due to the illegal capture of individuals, as long as the species has been abundant in the past, but not sought, otherwise the species may have always been scarce in these areas.

Keywords: Endangered birds, population abundance, forest use, Espinal and Monte.

\section{INTRODUCCIÓN}

El Cardenal amarillo (Gubernatrix cristata, Aves, Thraupidae) es endémico de América del Sur, y su área de distribución se extiende desde el extremo sur de Brasil hasta la República Argentina, e incluye a la República Oriental del Uruguay (Ridgely y Tudor, 1994; Azpiroz, 2012). En Argentina, su distribución comprende una amplia región desde el norte al centro del país, coincidiendo en general con la distribución de la Provincia Fitogeográfica del Espinal (Cabrera, 1976), donde habita en áreas con bosques abiertos y matorrales (Narosky e Izurieta, 2010). En la provincia de Buenos Aires se encuentra asociado a bosques de caldén (Prosopis caldenia), chañar (Geoffroea decorticans) y arbustales del sector sudoeste, principalmente en los partidos de Bahía Blanca, Puan, Villarino y Patagones, con predominio de especies como la jarilla (Larrea spp.), el piquillín (Condalia microphylla) y el alpataco (Prosopis alpataco), entre otras especies xerófilas (Narosky y Di Giácomo, 1993).

Las poblaciones del Cardenal amarillo han disminuido en forma alarmante debido a causas antrópicas, principalmente por la captura ilegal para el comercio como ave de jaula, y además por la pérdida y fragmentación de hábitat nativo; y también a agricultura, ganadería, extracción de madera, e incendios accidentales o intencionales (Collar et al., 1992; Pessino, 2001; Pessino y Tittarelli,
2006; SAyDS, 2007; Arab, Rovatti, Ttittare1li, 2015; Birdlife International, 2017). La preocupante situación de la especie evidenciada por el bajo número de individuos totales estimados en 1500 a 3000 individuos (Martins-Ferreira in litt, 2007) ha llevado a que se encuentre actualmente en el Apéndice II de la Convención sobre el Comercio Internacional para el Tráfico de Especies Sivestres (CITES), y en la categoría «En peligro» (Birdlife International 2018). En tanto, en Uruguay está incluida en la categoría de conservación «En Peligro» (Azpiroz, 2017), en Brasil es considerada amenazada (MMA, 2003) y «En Peligro» (Martins-Ferreira et al., 2013), y en Argentina está categorizada como «En peligro» (Secretaría de Ambiente y Desarrollo Sustentable de la Nación [SAyDS] y Aves Argentinas/Asociación Ornitológica del Plata [AA/AOP], 2017).

Dada la preocupante situación de conservación del Cardenal amarillo en Argentina, se vienen realizando esfuerzos en los últimos años por parte de diversos actores (organismos de gestión pública nacional y provinciales, organizaciones no gubernamentales, organismos de investigación, ornitólogos profesionales y aficionados) para obtener información actualizada de la especie, principalmente sobre distribución, abundancia, uso de hábitat, biología reproductiva, parasitismo y genética (Martins-Ferreira, Santos, Haddrath, Baker, Freitas, 2010; Domínguez, Reboreda, Mahler, 2015, 2016; Domínguez 
et al., 2017). A pesar de esto, el conocimiento general de la especie y del estado de sus poblaciones sigue siendo insuficiente, lo cual impide desarrollar planes de gestión eficientes para su conservación.

El objetivo de este trabajo es aportar al conocimiento del estado actual de las poblaciones de Cardenal amarillo en la provincia de Buenos Aires, actualizando y brindando nuevos datos sobre la distribución y abundancia de la especie en el sur de la provincia. También se realiza un aporte sobre el uso del bosque que realiza el Cardenal amarillo en los ambientes de Espinal y Monte en una de las áreas más australes de su distribución.

\section{MATERIALES Y MÉTODOS}

\section{ÁREA DE ESTUDIO}

El estudio se llevó a cabo en el sur de la provincia de Buenos Aires. Dicha área fue zonificada latitudinalmente en tres zonas: Zona Norte (sur del partido de Puan y norte del partido de Villarino); Zona Centro (centro y sur de Villarino, y norte del partido de Patagones); y Zona Sur (centro y sur de Patagones). Desde el punto de vista fitogeográfico, el norte del área de estudio (partidos de Puan, Villarino, y norte de Patagones) corresponde a la provincia del Espinal, distrito del Caldén, con una amplia transición hacia la provincia fitogeográfica del Monte en dirección suroeste (centro y sur de Patagones) (Cabrera, 1976; Burkart, Bárbaro, Sánchez, Gómez, 1999). Los límites entre estas provincias fitogeográficas son difíciles de establecer debido a que el Espinal forma extensos ecotonos hacia el oeste y sur con las ecorregiones Monte y Pampeana, donde coexisten especies de ambas regiones con la influencia de disturbios naturales y antrópicos. La fisonomía natural varía desde el bosque xerófilo hasta el matorral o estepa arbustiva xerófila (Torres Robles, Arturi, Contreras, Peter, Zeberio, 2015).

El distrito del Caldén, se caracteriza por la abundancia de caldén (Prosopis caldenia), que forma bosques xerófilos más o menos densos. Como árboles secundarios se encuentran el algarrobo negro (Prosopis ni- gra), el algarrobo dulce (Prosopis flexuosa), el chañar (Geoffroea decorticans), la sombra de toro (Jodina rhombifolia) y el molle (Schinus johnstonii). Entre los arbustos, son frecuentes el piquillín (Condalia microphylla), la tramontana (Ephedra triandra), el yaoyin (Lycium chilense), el alpataco (Prosopis alpataco), la pichanilla (Senna aphylla), el manca potrillo (Prosopidastrum globosum), el solupe (Ephedra ochreata) y la jarilla ( $L a-$ rrea divaricata). La provincia fitogeográfica del Monte se caracteriza por la dominancia de zigofiláceas arbustivas, especialmente jarillas (Larrea spp.), asociadas a arbustos del género Prosopis, junto con especies perennes no suculentas.

El clima del área es semiárido templado, con veranos cálidos e inviernos moderados, sin ningún exceso estacional de agua, con una temperatura media anual máxima de $22^{\circ} \mathrm{C}$ y mínima de $7^{\circ} \mathrm{C}$. La precipitación media anual es de $590 \mathrm{~mm}$ al norte de la región y $350 \mathrm{~mm}$ al sur, con máximos en otoño y primavera, y con una alta variabilidad interanual. Predominan los vientos secos del sector noroeste, oeste y norte, especialmente en primavera y verano. La altura del terreno no supera los 200 msnm (OTBN Buenos Aires 2014).

\section{Muestreo}

Se realizaron 11 campañas de muestreo entre los años 2015 y 2017, en las cuales se establecieron 13 transectas en los partidos de Puan, Villarino y Patagones. Se recorrieron $1.183 \mathrm{~km}$ de transectas, $265 \mathrm{~km}$ fueron repetidas (re-visitadas), y $918 \mathrm{~km}$ fueron transectas no repetidas; y se establecieron 303 puntos de muestreo (Tabla 1).

La búsqueda de ejemplares de Cardenal amarillo se realizó mediante un muestreo extensivo de puntos independientes de radio ilimitado con la utilización de «playback» (modificación del método propuesto por la ONG Aves Argentinas/Asociación Ornitológica del Plata). Estos puntos fueron ubicados sobre transectas predefinidas en gabinete mediante el análisis de imágenes satelitales en las que se evidenciaba la presencia de 
Tabla 1. Resultados por zona muestreada de la provincia de Buenos Aires.

\begin{tabular}{|c|c|c|c|c|c|c|c|c|c|c|}
\hline \multirow[t]{2}{*}{ Zona } & \multirow{2}{*}{$\begin{array}{l}\text { Longitud } \\
\text { total } \\
\text { transectas } \\
(\mathbf{k m})\end{array}$} & \multirow{2}{*}{$\begin{array}{l}\text { Longitud } \\
\text { parcial } \\
\text { transectas } \\
(\mathbf{k m})\end{array}$} & \multirow{2}{*}{$\begin{array}{l}\mathrm{N}^{\circ} \text { total de } \\
\text { puntos de } \\
\text { muestreo }\end{array}$} & \multirow{2}{*}{$\begin{array}{l}\mathrm{N}^{\circ} \text { total de } \\
\text { puntos } \\
\text { parciales } \\
\text { de } \\
\text { muestreo }^{\mathrm{b}}\end{array}$} & \multirow{2}{*}{$\begin{array}{l}\mathrm{N}^{\circ} \mathrm{de} \\
\text { registros }\end{array}$} & \multirow{2}{*}{$\begin{array}{l}\mathrm{N}^{\circ} \text { de } \\
\text { individuos } \\
\text { registrados }\end{array}$} & \multicolumn{4}{|c|}{ Abundancia relativa } \\
\hline & & & & & & & $\begin{array}{l}\text { IKA } \\
\text { total }\end{array}$ & $\begin{array}{l}\text { IKA } \\
\text { parcial' }\end{array}$ & $\begin{array}{l}\text { IPA } \\
\text { total }^{\mathrm{j}}\end{array}$ & $\begin{array}{l}\text { IPA } \\
\text { parcial }^{k}\end{array}$ \\
\hline Norte & 401 & 286 & 150 & 79 & $3^{c}$ & $7^{f}$ & 0,017 & 0,024 & 0,047 & 0,089 \\
\hline Centro & 405 & 255 & 86 & 50 & $1^{\mathrm{d}}$ & $1^{g}$ & 0,002 & 0,004 & 0,012 & 0,02 \\
\hline Sur & 377 & 377 & 67 & 67 & $15^{\mathrm{e}}$ & $40^{h}$ & 0,11 & 0,11 & 0,45 & 0,45 \\
\hline Total & 1.183 & 918 & 303 & 196 & 19 & 48 & 0,04 & 0,05 & 0,16 & 0,24 \\
\hline
\end{tabular}

a De transectas no repetidas.

b En transectas no replicadas.

c Un registro fuera de los puntos de playback.

d Registro sobre la transecta, fuera de los puntos de playback.

e Cuatro registros sobre la transecta, fuera de los puntos de playback.

f Dos individuos fuera de los puntos de playback.

g Un individuo registrado sobre la transecta, fuera de los puntos de playback.

h Diez individuos registrados sobre la transecta, fuera de los puntos de playback.

i Para este cálculo se utilizó el número de individuos registrados en la longitud parcial de transectas.

¡ Para este cálculo se utilizó el número de individuos registrados en los puntos de playback y el número de puntos totales de muestreo.

k Para este cálculo se utilizó el número de individuos registrados en los puntos de playback y el número de puntos parciales de muestreo.

hábitat disponible (parches de bosques y matorreles) (Fig. 1). Dichas transectas tuvieron una distancia mínima de separación de 1000 metros, y fueron establecidas sobre caminos rurales secundarios y recorridas en vehículos. Los puntos de muestreo se establecieron durante el recorrido de las transectas, a medida que observábamos ambientes aptos para la especie, es decir, parcelas con presencia de parches de bosques y arbustales. La distancia mínima entre los puntos de muestreo establecidos fue de 1000 metros. Cada punto de muestreo fue georreferenciado.

En cada punto de muestreo se reprodujo dos veces el canto de un cardenal macho de grabaciones provenientes de la provincia de Río Negro (facilitado por Aves Argentinas), y dos veces el canto de otro cardenal macho de una grabación proveniente de la provincia de Santa Fe (López-Lanús, 2010). Se utilizaron dos cantos diferentes con el fin de comprobar la probable respuesta diferencial de la especie a cada uno. Cada canto fue reproducido durante 1,5 minutos, espaciados por aproximadamente 30 segundos entre sí e intercalados. Con el fin de maximizar la pro- babilidad de observación de los ejemplares, uno de los observadores se ubicó dentro del ambiente de muestreo para la aplicación del «playback», mientras que otro observador se ubicó sobre el vehículo, en ambos casos utilizando binoculares de $8 \times 42$.

Los muestreos fueron realizados a principios de la temporada reproductiva: octubre de 2015, septiembre de 2016; septiembre, octubre y noviembre de 2017; y en marzo de 2016. Dichos muestreos fueron realizados en horarios entre el amanecer y el atardecer. Algunas de las transectas de las zonas Norte y Centro fueron repetidas interanualmente en forma total o parcial, mientras que ninguna de las transectas de la Zona Sur fue repetida. En las transectas repetidas siempre se establecieron nuevos puntos de muestreo. Las campañas de muestreo duraron entre 1 y 3 días y cada transecta fue recorrida sólo una vez en cada visita. En cada día de muestreo se recorrieron entre 1 y 4 transectas dependiendo de la longitud de las mismas, de la distancia entre ellas, y de la cantidad de vehículos utilizados en simultáneo (máximo 2). 


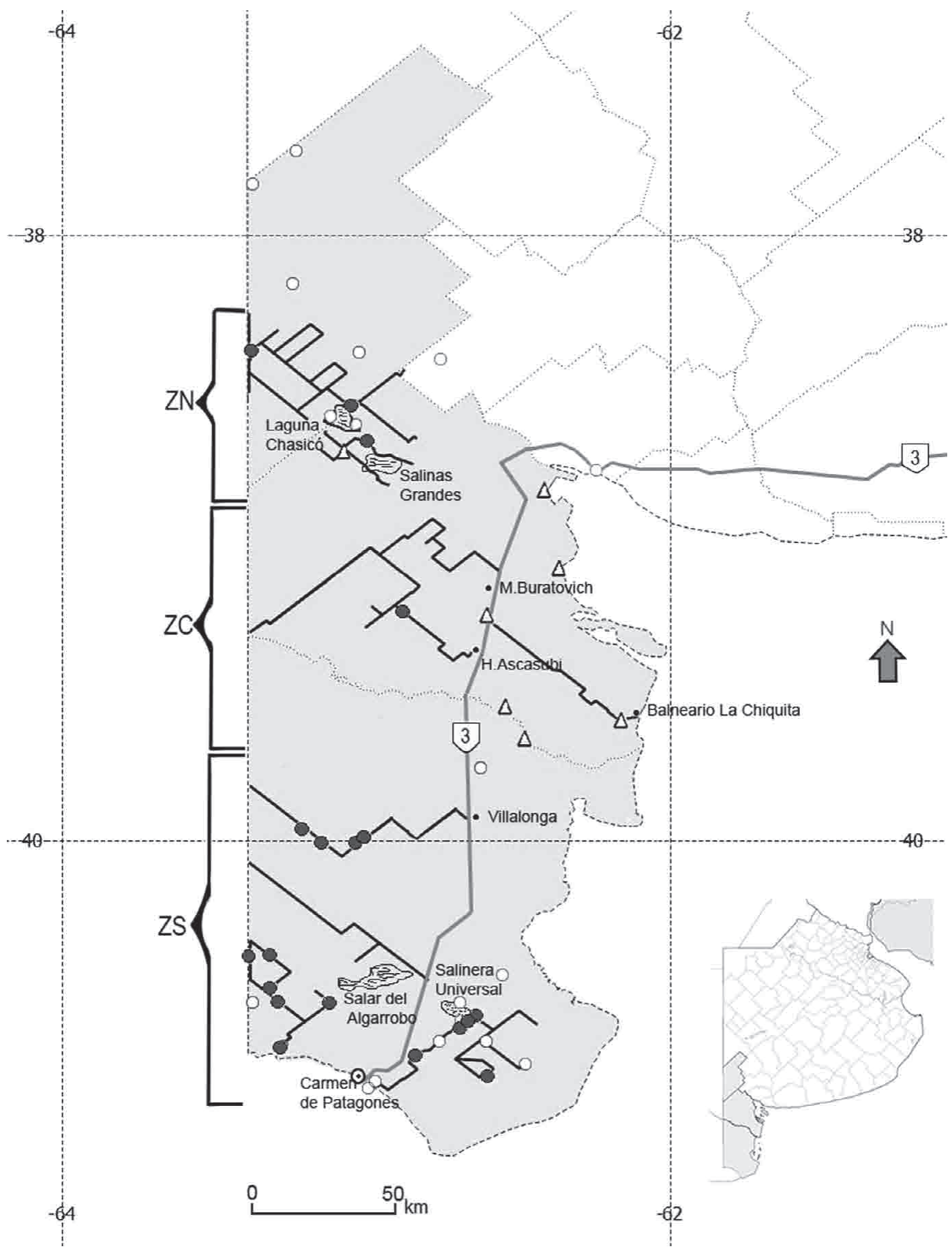

Figura 1. Sitios con registros de Cardenal amarillo en el sur de la provincia de Buenos Aires. De norte a sur se destacan los partidos de Puan, Villarino y Patagones (sombreado gris). Referencias: ZN = Zona Norte; ZC = Zona Centro; ZS= Zona Sur; $\bullet=$ registros de este estudio; $\Delta$ = registros 2008 - 2018 (Petracci y Sotelo, 2013; eBird, 2017; EcoRegistros, 2017; SAyDS, 2017) (el punto de los alrededores de la laguna Chasicó pertenece a dos registros contiguos); $\mathrm{O}=$ registros anteriores a 2008 (Verniere, 2004; MAyDS, 2017); - = transectas. 
Además del método sistemático de muestreo de puntos, las transectas fueron recorridas a baja velocidad (menos de $40 \mathrm{~km} / \mathrm{h}$ ) entre cada punto con la finalidad de detectar ejemplares de la especie también por fuera de los puntos de muestreo. Cuando se registraron individuos con este método, se contó el número de individuos y se determinó el sexo de cada uno, usando «playback» cuando fue necesario. Se consideró como registro a la detección de uno o más individuos de Cardenal amarillo en un punto sistemático de muestreo y a los individuos detectados fuera del muestreo sistemático.

En cada punto de muestreo se caracterizó la vegetación según el porcentaje de cobertura en un radio de 100 metros, diferenciando entre cobertura arbórea, cobertura arbustiva y cobertura herbácea. Se consideró como estrato arbóreo a la fracción dentro de un punto de muestreo integrado por especies leñosas cuya altura superase los 3 metros. En tanto que el estrato arbustivo fue definido como aquel conformado por especies leñosas con una altura igual o inferior a los 3 metros de altura.

\section{ANÁLISIS DE DATOS}

\section{REGISTROS Y ABUNDANCIA \\ DE CARDENAL AMARILLO}

La abundancia relativa del Cardenal amarillo fue estimada con dos índices: Índice Kilométrico de Abundancia (IKA) (número de individuos/km de transecta) e Índice Puntual de Abundancia (IPA) (número de individuos/punto de muestreo). Para el cálculo del IKA fueron utilizados los registros dentro del muestreo sistemático (puntos de muestreo con «playback») y los registros obtenidos por fuera de los puntos de muestreo durante el recorrido de las transectas. Para el cálculo del IPA sólo se consideró el número de individuos registrado en los puntos de muestreo con «playback».

\section{DisPonibiLIDAD Y USO DEL BOSQUE}

Para el análisis de datos de disponibilidad y uso del bosque fueron utilizados todos los puntos de las transectas que fueron recorri- das una sola vez, mientras que de aquellas transectas relevadas más de una vez (repetidas) se utilizaron los puntos muestreados en sólo una de las repeticiones temporales. El criterio utilizado fue el de seleccionar aquella visita con la mayor cantidad de puntos muestreados y/o que tuviera registros de Cardenal amarillo.

Para evaluar la variación en la cobertura del bosque entre los sitios utilizados y no utilizados por la especie, se realizó un Análisis de Kruskal-Wallis (K-W, criterio de rechazo en $\mathrm{p} \leq 0,05$ ). Se utilizó como variable dependiente el porcentaje de cobertura de bosque (árboles más arbustos), y como variable de clasificación el uso/no uso del bosque (sitios con registros versus sitios sin registros de Cardenal amarillo). También se realizó un análisis de Kruskal-Wallis (K-W) para explorar la variación en la cobertura por estratos (árboles y arbustos) y de la cobertura total del bosque (árboles más arbustos) entre las zonas Norte, Centro y Sur. Los análisis fueron realizados con el paquete estadístico Infostat (Di Rienzo et al., 2008).

\section{RESULTADOS}

\section{DISTRIBUCIÓN DE REGISTROS \\ Y ABUNDANCIA DE CARDENALES}

Se obtuvieron 19 registros del Cardenal amarillo con un total de 48 individuos observados (Tabla 1 y Apéndice 1, Fig. 1). Del total de registros, 13 ( $n=35$ individuos) fueron obtenidos en los muestreos sistemáticos de puntos, y 6 ( $\mathrm{n}=13$ individuos) dentro de las transectas pero fuera de los puntos de «playback». El valor promedio de individuos por punto positivo de registro fue de $2,53 \pm$ 0,60 (mín $=1$, máx $=4$ individuos, se indica desvío estándar). Se registraron 24 machos (16 adultos y 8 subadultos), 17 hembras, y 7 individuos en los que no se pudo determinar el sexo. El 78,9 \% de los registros y el 83,3 $\%$ de los individuos fueron observados en la Zona Sur; el 15,8 \% de los registros y el $14,6 \%$ de los individuos fueron en la Zona Norte; y el 5,3\% de los registros y el 2,1\% de los individuos fueron en la Zona Centro (Tabla 1 y Fig. 1). 
La abundancia de Cardenal amarillo fue más alta en la Zona Sur que en las Zonas Norte y Centro. Esto se ve reflejado en los valores de IKA parcial, en donde la Zona Sur tuvo 4,6 y 27,5 veces más que las Zonas Norte y Centro, respectivamente; y también en los valores de IPA parcial en donde la Zona Sur tuvo 5,06 y 22,5 veces más que las Zonas Norte y Centro, respectivamente (Tabla 1).

El $46 \%$ de los registros en los puntos de muestreo fueron obtenidos con la emisión del primer «playback», el $15 \%$ se obtuvieron con el segundo «playback», el $15 \%$ con el tercero, y el $23 \%$ con el cuarto. El 62\% de los registros ocurrieron con el canto proveniente de la provincia de Río Negro, mientras que el 38\% restante ocurrió con el canto proveniente de la provincia de Santa Fe.

\section{DISPONIBILIDAD \\ Y USO DEL BOSQUE}

No se hallaron diferencias estadísticamente significativas en la cobertura del bosque entre los sitios con registros de Cardenal amarillo y los sitios sin registros de la especie $(\mathrm{H}=0,02, \mathrm{p}=0,89$, test de K-W) (Fig. 2a).

Si bien no se encontraron diferencias significativas en la cobertura total del bosque entre las zonas analizadas (Norte, Centro y Sur) $(H=4,2, p=0,1199$, test de K-W $)$, la cobertura de árboles fue significativamente mayor en la Zona Norte y menor en la Zona Sur $(\mathrm{H}=84,04, \mathrm{p}<0,0001)$, mientras que la cobertura arbustiva fue significativamente mayor en la Zona Sur y menor en la Zona Norte $(H=63,98, p<0,0001)$ (Fig. 2b).

\section{DISCUSIÓN}

\section{DISTRIBUCIÓN DE REGISTROS Y ABUNDANCIA}

La distribución y abundancia de Cardenal amarillo en el sur de la provincia de Buenos Aires no es homogénea. Los resultados del presente estudio muestran que la mayor abundancia de Cardenal amarillo se encuentra en la Zona Sur, mientras que en las Zonas Norte y Centro la especie se presenta con menor frecuencia.

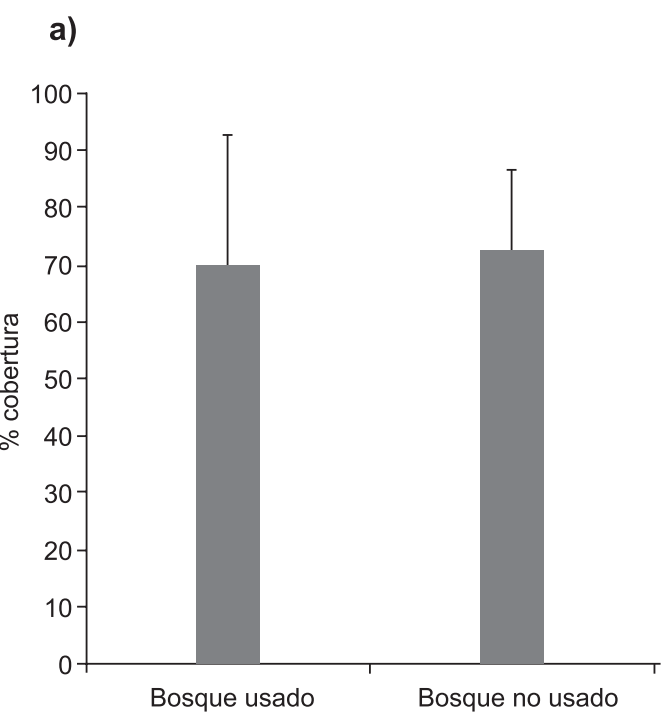

b)

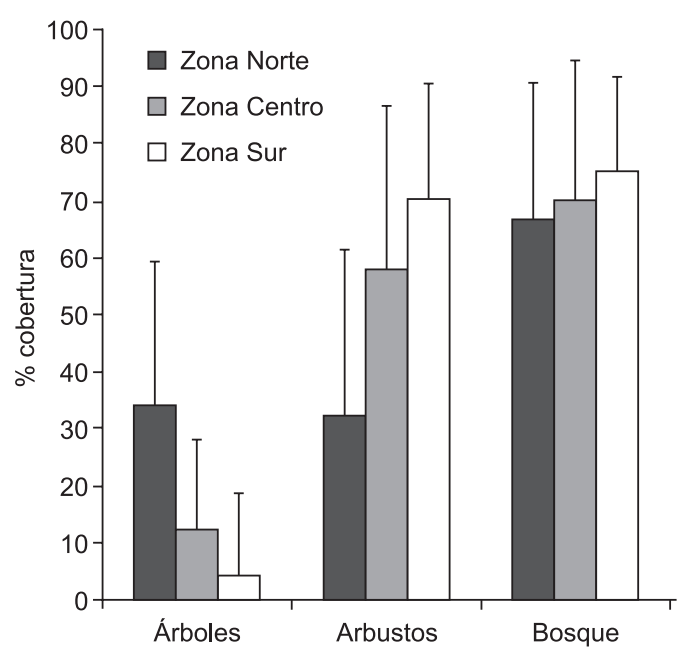

Figura 2. a) Porcentaje de cobertura (promedio \pm DS) de bosque (estrato arbóreo + estrato arbustivo) usado vs. no usado por el Cardenal amarillo en toda el área de estudio. b) Porcentaje de cobertura (promedio \pm DS) de los estratos arbóreo, arbustivo y de bosque [arbóreo + arbustivo] en las zonas Norte, Centro y Sur.

En la Zona Centro, hacia el oeste de la ruta 3 , aunque se realizó más de una campaña de muestreo, sólo se registró un individuo, lo que concuerda con la ausencia de registros publicados en dicha zona. Hacia el este de la ruta 3 existen 5 registros de al 
menos 18 individuos en los últimos 10 años, y 3 de ellos en los últimos 3 años (eBird, 2017; EcoRegistros, 2017; SAyDS, 2017). Sin embargo, en el presente estudio no se detectaron individuos de Cardenal amarillo en dicha zona, lo cual podría deberse a que la transecta fue recorrida una sóla vez y sobre un camino muy transitado, con el bosque muy degradado, fragmentado, o ausente, por uso agrícola.

En la Zona Norte (sur de Puan y norte de Villarino), en los alrededores de la Laguna Chasicó y las Salinas Grandes, la abundancia de Cardenal amarillo también es escasa, aunque mayor que en la Zona Centro, concordando con los escasos registros existentes en los últimos años (eBird, 2017; SAyDS, 2017). Esto podría explicarse por el mayor esfuerzo de muestreo realizado en la Zona Norte (150 puntos versus 86 puntos).

Resulta difícil establecer si la especie presentó en el pasado mayor abundancia en la Zona Norte, particularmente en los alrededores de la Laguna Chasicó, o si siempre ha sido una especie escasa en la zona tal como lo muestra este estudio. En el año 1967 se realizaron 3 campañas de muestreo en las inmediaciones de la Laguna Chasicó, en las cuales fueron colectados 7 individuos (SAyDS, 2017); mientras que desde 2015 a 2017 se obtuvieron sólo 4 nuevos registros ( $\mathrm{n}=8$ individuos) (SAyDS, 2017; y este estudio), con un alto esfuerzo de muestreo (ver Anexo). Algo más al norte $(20 \mathrm{~km}$ de la Laguna Chasicó), en zonas en donde aún existe una buena cobertura boscosa, se cuenta con un registro de 6 individuos en 2005 (eBird 2017), y un registro de 3 individuos a aproximadamente $30 \mathrm{~km}$ al noroeste de dicha laguna (este estudio). La escasez de registros previos a este trabajo y los pocos registros de este estudio parecen indicar que la especie siempre ha sido escasa en la zona, aunque desconocemos si el esfuerzo de muestreo en el pasado fue similar al realizado en el presente estudio.

En la Zona Sur se obtuvieron numerosos registros novedosos para la especie, principalmente al oeste de la ruta 3 , donde existía un solo registro de 2 individuos en 2005
(SAyDS, 2017). Por otra parte, confirmamos la alta abundancia relativa al sureste de Patagones, sitio donde existen varios registros históricos, la mayoría previos a la década de 1990 (SAyDS, 2017). Se confirma la presencia de la especie y se observa una abundancia relativamente alta en los alrededores de la Salinera Universal, sitio donde solo existe un ejemplar colectado en 2003 (SAyDS, 2017). El noreste de la Zona Sur (noreste del partido de Patagones) se encuentra actualmente muy modificado debido al uso agrícola-ganadero, por lo tanto no fue relevado en este estudio, aunque sólo existe un registro histórico (año 1962), de 2 individuos, para el área. Los datos obtenidos en el presente estudio confirman y aumentan los registros en la Zona Sur.

En el sur de la provincia de Buenos Aires existen áreas no relevadas en este estudio que revisten interés, como por ejemplo, el norte y centro de Puan, ya que allí existen registros históricos de la especie, incluso con registros de nidificación (Narosky, Di Giacomo, López Lanús, 1990; SAyDS, 2017).

\section{DISPONIBILIDAD Y USO DEL BOSQUE}

A la fecha, no existe bibliografía disponible que brinde información sobre el uso y selección de hábitat que realiza la especie. Los escasos estudios realizados sobre su biología reproductiva indican que construyen nidos tanto en árboles hasta los 6,5 $\mathrm{m}$ de altura (Domínguez, 2015; Domínguez, Ayarragaray, Lapido, 2015), como en arbustos (de la Peña, 2013). En los sitios relevados en las zonas Norte, Centro y Sur la relación de porcentajes de cobertura de árboles/arbustos disminuye, notándose un gradiente latitudinal. La escasez de Cardenal amarillo en las zonas Norte y Centro en relación a la abundancia registrada en la Zona Sur no podría, en principio, ser atribuida a las diferencias halladas en la disponibilidad de cobertura arbórea y arbustiva.

La baja abundancia de Cardenal amarillo en el norte y centro de la región estudiada, concuerda con la ausencia de datos históricos de abundancia, tanto para el oeste de 
la Zona Centro (provincia de Buenos Aires), sureste de la provincia de La Pampa y el noreste de la provincia de Rio Negro (eBird, 2017; SAyDS, 2017). Debido a la ausencia de información publicada que permita conocer el esfuerzo de muestreo y los métodos utilizados para registrar la especie en el pasado, en las mencionadas zonas, se proponen dos hipótesis. Por un lado, la especie podría haber sido buscada con un esfuerzo de muestreo alto y no haber sido hallada, tal como ocurrió en este estudio. Por otro lado podría esperarse que, no siendo intensamente buscada, la especie fuera más abundante en épocas pasadas. Si esto último fuera así, la captura ilegal de ejemplares podría ser el principal factor de retracción y disminución de la especie en esta área, tal como ocurre en toda su área de distribución (Birdlife International, 2017).

Resulta necesario realizar a futuro estudios específicos sobre disponibilidad y selección del hábitat de la especie, tanto a escala regional, de paisaje, como de microhábitat, para conocer la relación entre la distribución y abundancia de la especie y el estado de conservación del Espinal y Monte en el área de distribución más austral del Cardenal amarillo. Por otra parte, la generación de nuevas herramientas de gestión y conservación, u optimización de las ya existentes, conjuntamente con el bosque, resultan poco factibles y eficaces si no se cuenta con información ecológica adecuada.

El presente trabajo aporta nueva información sobre la abundancia y distribución del Cardenal amarillo en la provincia de Buenos Aires y confirma y actualiza parte de la información existente. Es importante la conservación del bosque en sentido amplio (Espinal y Monte), incluyendo áreas arbustivas, además de la optimización de los esfuerzos de fiscalización y concientización sobre la captura ilegal, para la conservación de la especie en la provincia de Buenos Aires.

\section{AGRADECIMIENTOS}

A Javier Roig de la Dirección de Áreas Naturales Protegidas - OPDS, por la realización de los mapas. A Juan Serwatowski, de la Dirección de Recursos Naturales - OPDS, por su participación y asistencia en el campo en campañas de muestreo. A Aníbal Areco y Marcos Suárez, de la Dirección de Áreas Naturales Protegidas - OPDS, y a Rodolfo Sarria, por sus participaciones en diferentes campañas de muestreo. A Diego Gutiérrez, del Departamento de Automotores - OPDS. A Mauro Massi del CORFO Ascasubi, por la hospitalidad brindada durante parte de las campañas de muestreo. A Aves Argentinas/Asociación Ornitológica del Plata por facilitar el canto del Cardenal amarillo de la provincia de Río Negro. A la Secretaría de Ambiente y Desarrollo Sustentable de la Nación por la facilitación del banco de registros del Cardenal amarillo. A eBird por facilitar los registros de Cardenal amarillo para la provincia de Buenos Aires. A tres revisores anónimos por la lectura crítica y las correcciones y sugerencias aportadas al manuscrito.

\section{FINANCIAMIENTO}

Este trabajo fue posible gracias al financiamiento y el apoyo logístico del Organismo Provincial para el Desarrollo Sostenible de la provincia de Buenos Aires (OPDS).

\section{PARTICIPACIÓN}

Germán Marateo y Diego Archuby: muestreos de campo, análisis de datos y redacción del artículo. Fabrizio Piantanida, Martín Sotelo y Fernando Segura: muestreos de campo y revisión del artículo.

\section{CONFLICTOS DE INTERÉS}

No existen conflictos de interés entre los autores ni con terceros.

\section{LITERATURA CITADA}

Arab M.F., Rovatti G., Tittarelli R.F. (2015). Mascotismo de aves silvestres en Santa Rosa, La Pampa. Nuestras Aves, 60, 106-108.

Azpiroz A.B. (2012). Aves de las Pampas y Campos de Argentina, Brasil y Uruguay. Una guía de identificación. Pressur, Nueva Helvecia, Uruguay. 
Azpiroz A.B. (2017). Cardenal amarillo (Gubernatrix cristata]. En Libro Rojo de las Aves de Uruguay. Biología y conservación de las aves en peligro de extinción a nivel nacional. Categorías "Extinto a Nivel Regional", En "Peligro Crítico", En "Peligro" (173-188). Azpiroz A.B., Jlmenez S., Alfaro M. (eds). DINAMA Y DINARA, Montevideo.

Birdlife International (2018). Species factsheet: Gubernatrix cristata. Descargado de http://www.birdlife.org el $17 / 10 / 2018$.

Burkart R., Bárbaro N.O., Sánchez R.O., Gómez D.A. (1999). Ecorregiones de la Argentina. Administración de Parques Nacionales, Buenos Aires.

Cabrera A.L. (1976). Regiones fitogeográficas Argentinas. Enciclopedia Argentina de Agricultura y Jardinería, Segunda Edicion, Vol II. Buenos Aires.

Collar N.J., Gonzaga L.P., Krabbe N., Madrono Nieto A., Naranjo L.G., Parker T.A., Wege D.C. (1992). Threatened Birds of the Americas: the ICBP/IUCN Red Data Book. Cambridge: International Council for Bird Preservation.

de la Peña, M.R. (2013). Nidos y reproducción de las aves argentinas. Ediciones Biológica. Serie Naturaleza, Conservación y Sociedad No 8. Santa Fe, Argentina. $590 \mathrm{pp}$.

Di Rienzo J.A., Casanoves F., Balzarini M.G., Gonzalez L., Tablada M., Robledo C.W. (2008). InfoStat, versión 2008. Grupo InfoStat, FCA, Universidad Nacional de Córdoba, Argentina.

Domínguez M. (2015). Estructuración poblacional del Cardenal Amarillo (Gubernatrix cristata): implicancias para su conservación. Tesis doctoral, Facultad de Ciencias Exactas y Naturales, Universidad de Buenos Aires, $133 \mathrm{pp}$.

Domínguez M., Ayarragaray M., Lapido R. (2015). Nidos de Cardenal amarillo (Gubernatrix cristatal en la provincia de San Luis, Argentina. Nuestras Aves, 60, 8-10.

Domínguez M., Reboreda J.C., Mahler B. (2015). Impact of Shiny Cowbird and botfly parasitism on the reproductive success of the globally endangered YelIow Cardinal Gubernatrix cristata. Bird Conservation International, 25, 294305.

Domínguez M., Reboreda J.C., Malher B. [2016). Effects of fragmentation and hybridization on geographical patterns of song variation in the endangered Yellow Cardinal Gubernatrix cristata. Ibis, 158, 738-746.
Domínguez M., Tiedemann R., Reboreda J.C., Segura L., Tittarelli F., Mahler B. (2017). Genetic structure reveals management units for the Yellow Cardinal (Gubernatrix cristata), endangered by habitat loss and ilegal trapping. Conservation Genetics, 18, 1131-1140.

eBird Basic Dataset. Version: EBD_relMay2017. Cornell Lab. of Ornithology, Ithaca, New York. May 2017.

EcoRegistros (2017). Cardenal Amarillo (Gubernatrix cristata] - Ficha de la especie. Accedido de http://www.ecoregistros. org el 11/12/2017.

Fosberg F.R. (1961). Classification of vegetation for general purposes. Tropical Ecology, 2, 1-28.

López-Lanús B. (2010). DVD de Sonidos de Aves de Argentina y Uruguay. En Aves de Argentina y Uruguay, guía de identificación, edición total. Narosky S., Izurieta D. Vázquez Mazzini eds.

Martins-Ferreira C., Santos M.O., Haddrath O., Baker A.J., Freitas T.R.O. (2010). Isolation and characterization of $10 \mathrm{mi}-$ crosatellite loci in the Yellow Cardinal Gubernatrix cristata. Molecular Ecology Resources, 10, 751-754.

Martins-Ferreira C., Repenning M., Vargas Damiani R. (2013). Gubernatrix cristata. En Serafini P.P. (org.). Plano de Ação Nacional para la conservação dos paseriformes ameaçados dos Campos Sulinos y Espinilho. Série Espécies Ameaçadas, 31 (116-119). Instituto Chico Mendes de Conservação da Biodiversidade, ICMBio, Brasilia.

MMA (2003). Lista Nacional das Espécies da Fauna Brasileira Ameaçada de Extinção. Anexo à Instrução Normativa no. 3 do MMA, de 25/05/2003, publicada no Diário Ofical da União no. 101, de 28/05/2003, Seção 1, p. 88.

Narosky S., Di Giacomo A.G., Lopez-Lanus B. (1990). Notas sobre aves del sur de Buenos Aires. Hornero, 13, 173-178.

Narosky S., Di Giacomo A.G. (1993). Las aves de la provincia de Buenos Aires: Distribución y estatus. Asociación Ornitológica del Plata, Vázquez Mazzini y L.O.L.A. eds.

Narosky S., Izurieta D. (2010). Aves de Argentina y Uruguay, guía de identificación, edición total. Vázquez Mazzini eds.

OTBN (Ordenamiento Territorial de Bosques Nativos, Provincia de Buenos Aires] (2014). Dirección de Recursos Naturales, Organismo Provincial para el Desarrollo Sostenible. Documento Técnico Inédito. Buenos Aires, Argentina. 
Pessino M. (2001). Cardenal amarillo (Gubernatrix cristata). Diagnóstico sobre la situación de la especie en la provincia de La Pampa y sudoeste de la provincia de Buenos Aires. Dirección de Fauna y Flora Silvestres (Secretaría de Recursos Naturales y Ambiente Humano]. Dirección de Recursos Naturales de la provincia de La Pampa, Santa Rosa.

Pessino M., Tittarelli R.F. (2006). The Yellow Cardinal (Gubernatrix cristata): a diagnosis of its situation in the province of La Pampa, Argentina. Gestión Ambiental, 12, 69-76.

Petracci P., Sotelo M. (2013). Aves del Estuario de Bahía Blanca. Una herramienta para su conocimiento y conservación. Muelle Sur, 196 pp.

Ridgely R.S., Tudor G. (1994). The birds of South America. Volume I: The Oscine Passerines. University of Texas Press, Austin.

SAyDS (Secretaría de Ambiente y Desarrollo Sustentable de la Nación) (2007). Primer inventario nacional de bosques nativos. Inventario de campo de la región del Espinal, Distritos Caldén y Ñandubay. Proyectos Bosques Nativos y Áreas Protegidas, BIRF 4085-AR, 1998-2001
República Argentina. Informe Regional Espinal, segunda etapa.

SAyDS (Secretaría de Ambiente Y Desarrollo Sustentable de la Nación) (2017). Banco de registros del Cardenal amarillo. Dirección de Fauna Silvestre y Conservación de la Biodiversidad, SAyDS. Buenos Aires, Argentina.

SAyDS y AA (Secretaría de Ambiente y Desarrollo Sustentable de la Nación - Aves Argentina/Asociación Ornitológica del Plata) (2017). Categorización de las Aves de la Argentina (2015). Informe del Ministerio de Ambiente y Desarrollo Sustentable de la Nación y de Aves Argentinas, edición electrónica. C.A. Buenos Aires, Argentina. 147 pp.

Torres Robles S.S., Arturi M.F., Contreras C., Peter G., Zeberio J.M. (2015). Variaciones geográficas de la estructura y composición de la vegetación leñosa en el límite entre el espinal y el monte en el Noreste de la Patagonia (Argentina). Bol. Soc. Argent. Bot. 50, 209-215.

Verniere L.C. (2004). Comunidades de aves asociadas a relictos de vegetación natural en la costa del partido de Bahía Blanca. Tesis de Licenciatura, Universidad Nacional del Sur, Bahía Blanca. 37 pp. 
Registros fotográficos.

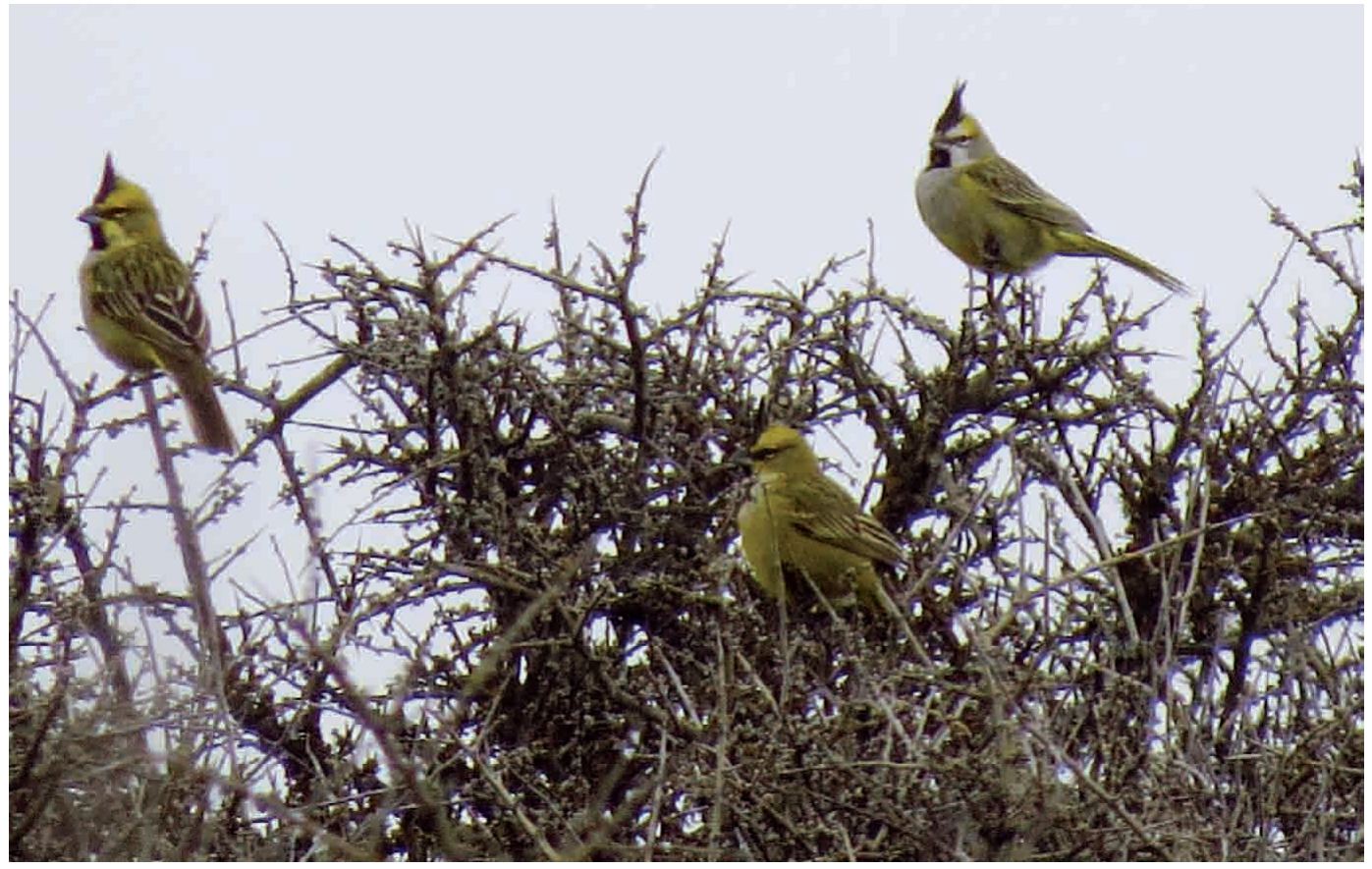

Figura 1. Dos machos y una hembra registrados el 15/10/15 en el partido de Patagones (fotografía: F. Piantanida).

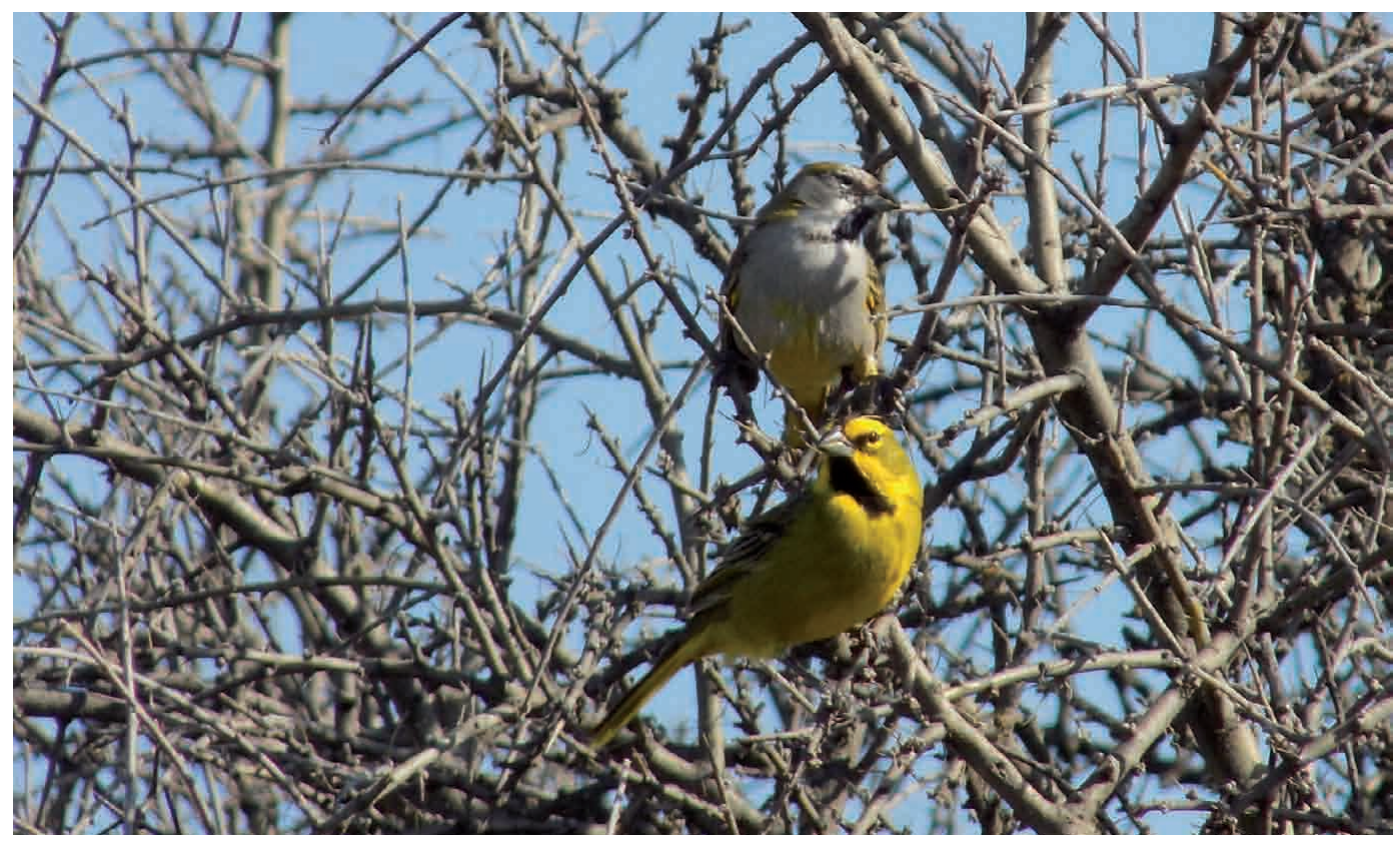

Figura 2. Pareja registrada (1 macho y 1 hembra) el 19/9/17 en el partido de Patagones (fotografía: D. Archuby). 


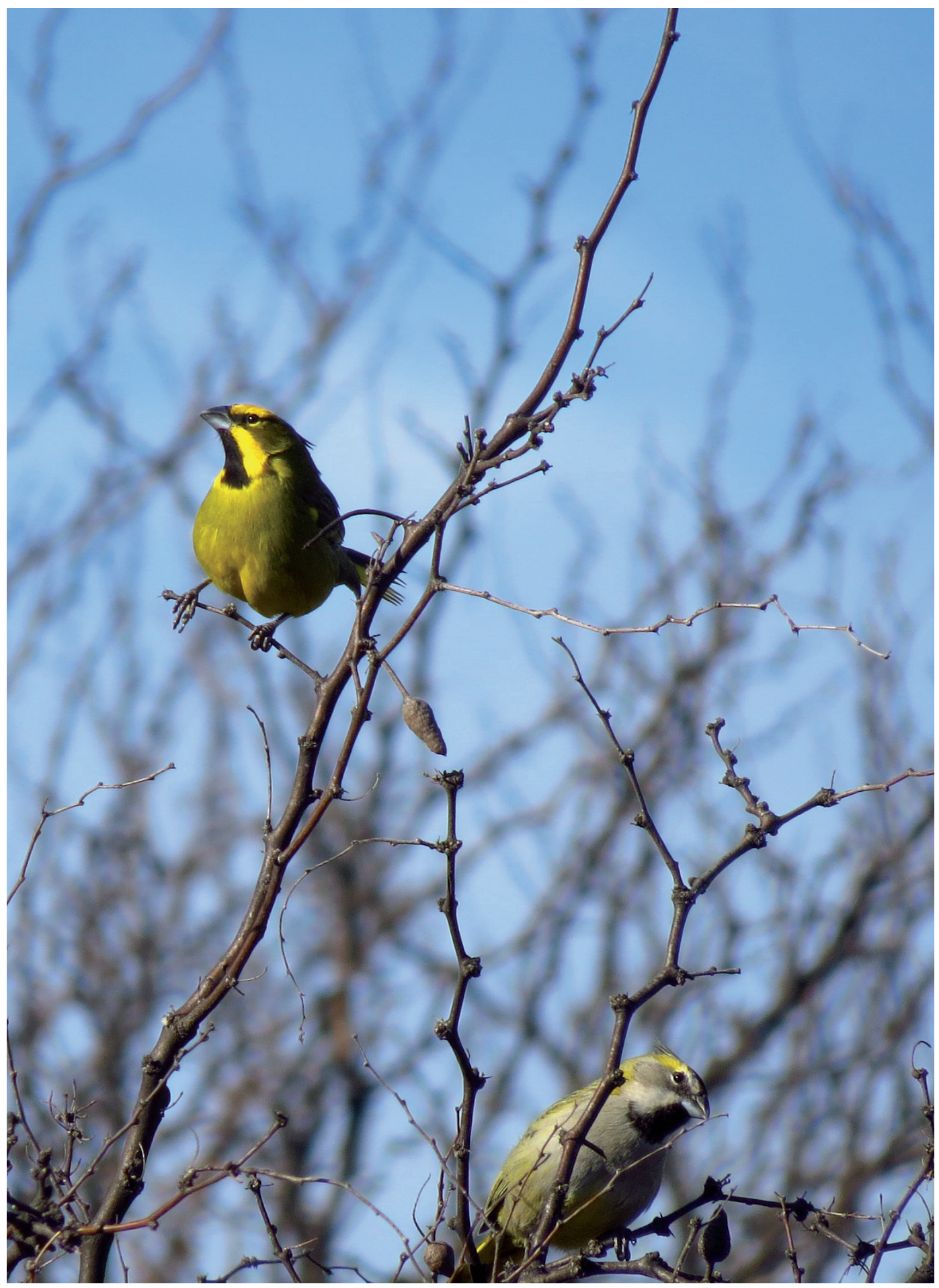

Figura 3. Pareja registrada ( 1 macho y 1 hembra) el 20/9/17 en la Reserva Chasicó, partido de Villarino (fotografía: F. Piantanida). 